\title{
Effects of Molar Ratios of Thiol-Ene Monomers on the Morphology and Electro-Optical Properties of Polymer Dispersed Liquid Crystal Films
}

\author{
Su-Cheol Park ${ }^{1}$, Jung-Dae Cho ${ }^{1}$, Jin-Who Hong ${ }^{2 *}$ \\ ${ }^{1}$ Institute of Photonics \& Surface Treatment, Q-Sys Co. Ltd., Gwangju, South Korea \\ ${ }^{2}$ Department of Polymer Science \& Engineering, Chosun University, Gwangju, South Korea \\ Email: *jhhong@chosun.ac.kr
}

Received August 8, 2013; revised September 8, 2013; accepted September 15, 2013

Copyright (C) 2013 Su-Cheol Park et al. This is an open access article distributed under the Creative Commons Attribution License, which permits unrestricted use, distribution, and reproduction in any medium, provided the original work is properly cited.

\begin{abstract}
We synthesized tetrafunctional allyl ether monomer (4-AE) and investigated the effects of the different molar ratios of trimethylolpropane tris-(3-mercaptopropionate) (3-SH) to 4-AE on the photopolymerization behavior, morphology, and electro-optical properties of thiol-ene-based PDLC films. Photo-DSC and DSC analyses revealed that the PDLC sample containing $45 \mathrm{wt} \% 3-\mathrm{SH}$ and $45 \mathrm{wt} \%$ 4-AE gave the highest exotherm, the fastest photocure rate, and the highest $T_{\mathrm{g}}$ due to the matched stoichiometry. Morphological observations and electro-optical measurements showed that the PDLC sample with the matched molar ratio gave the smallest LC droplet size, the highest threshold, driving voltages, and lowest saturation transmittance because the orientation of LC molecules got difficulty in small droplets. The stoichiometric ratios of 3-SH to 4-AE played an important role in controlling the photocure rate, phase separation rate, microstructures of LC droplets, and electro-optical properties of thiol-ene-based PDLC systems.
\end{abstract}

Keywords: Photopolymerization; PDLC; Thiol-Ene; Stoichiometry; Driving Voltage

\section{Introduction}

Polymer dispersed liquid crystal (PDLC) is a composite containing low-molecular-weight liquid crystal (LC) domains randomly dispersed within a continuous polymer matrix. A thin $(5-25 \mu \mathrm{m})$ film between transparent conducting substrates can be switched between an intensely scattering state and a transparent state by applying an electric field across the film. In the field-off (OFF) state, the incident light is scattered due to the mismatch in the refractive indices of the randomly oriented liquid crystal molecules and the polymer matrix. Under an electric field, the LC directors are oriented in the direction of the electric field within the LC domains, which matches the refractive index of the polymer matrix to that of the ordinary component of the LC, and thus, the PDLC film appears transparent. These attractive intrinsic electrooptical characteristics mean that the PDLC can be used as a switchable glass, an optical devices, and a display devices [1-6].

A large number of researches have performed since the early 1990s which address the phase separation process,

\footnotetext{
"Corresponding author.
}

morphology, and electro-optical properties of PDLC systems based on photopolymerization processes. Thiol-ene and acrylate systems have been the major photopolymerization processes used to produce PDLC films. Thiolene systems polymerize via the step-growth polymerization mechanism, whereas acrylate systems polymerize via the chain-growth radical polymerization mechanism [7-13].

In the step-growth polymerization, the increase in molecular weight is much slower and the gelation takes place at a higher degree of monomer conversion. These intrinsic properties of thiol-ene systems enable the PDLC films to be produced with a uniform morphology with narrow dispersity in the shape and size of LC droplets and a higher degree of LC phase separation. The thiolene system used most widely to construct PDLC films is commercial Norland Optical Adhesive (NOA65). The refractive index of cured NOA65 is $1.50-1.52$, which corresponds to the normal range of refractive indices of conventional nematic LC mixtures consisting of cyanobiphenyl molecules, which yields PDLC films with good electro-optical performance [7,10,14-16].

One of the most important factors determining the 
electro-optical properties of PDLC is its morphology which is strongly affected by the prepolymer composition, solubility between the LC and prepolymer, the polymerization kinetics, and the phase separation mechanism [17-27]. For the PDLC films prepared using thiolene systems, the droplet morphology and structural parameters such as droplet size, shape, distribution and density can be varied with the chemical characteristics of the prepolymer and polymerization kinetics. Therefore, it is important to elucidate how the molar ratios of thiol-ene monomers affect their morphology and electro-optical properties.

While the morphology and electro-optical properties of thiol-ene-based PDLC films have been examined extensively, systematic approaches to the prepolymer compositions have been very limited because most studies have used a formulated commercial product (NOA65) as the prepolymer. It is important to consider variations in the kinetics and network structure of thiol-ene systems according to the molar ratio of thiol-ene monomers. In this study, we investigated the effects of the molar ratio of thiol-ene monomers on the photopolymerization-induced phase separation process, morphology, and PDLC performances.

\section{Experimental}

\subsection{Materials and Sample Preparation}

Tetrafunctional allyl ether monomer (4-AE) was prepared from trimethylolpropane diallyl ether (2-AE) and isophorone diisocyanate. The synthesis scheme is shown in Figure 1. Trimethylolpropane tris-(3-mercaptopropionate) (3-SH) was used as the thiol monomer. The monomers were purchased from Aldrich Chemical and used without further purification. 2,2-Dimethoxy-2-phenylacetophenone (Irgacure 651, Ciba Specialty Chemicals) was used as the photoinitiator. A multicomponent eutectic LC

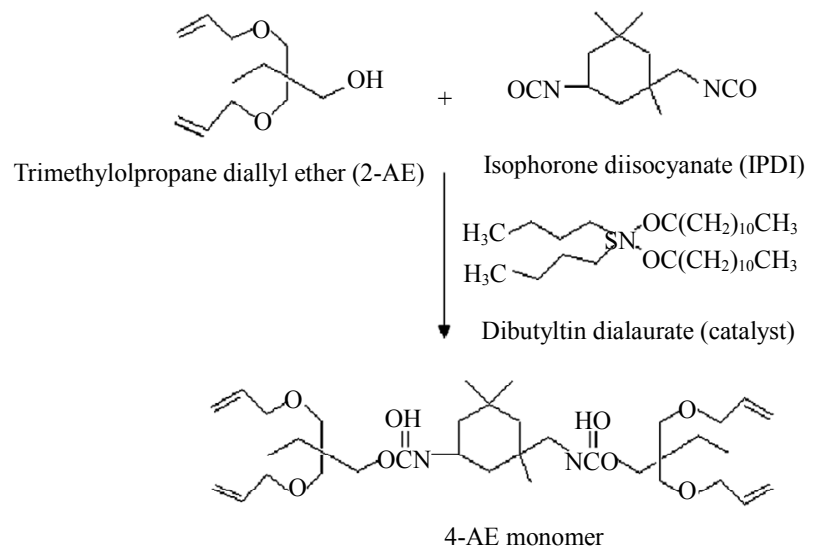

Figure 1. Synthesis of tetrafunctional allyl ether monomer (4-AE) from trimethylolpropane diallyl ether (2-AE) and isophorone diisocyanate. mixture of cyanobiphenyls (E7, Merck) was used as a nematic LC. The mixtures for the PDLC films were prepared by directly mixing the E7 and the formulated prepolymer at a 1:1 weight ratio over $5 \mathrm{~h}$ at room temperature; these were used as the initial reactive mixtures for UV curing. The PDLC films were prepared by sandwiching the initial reactive mixtures between two conductive ITO-coated PET films $\left(10 \times 10 \mathrm{~cm}^{2}\right)$. The film gap was adjusted to $20 \mu \mathrm{m}$ by using polymer ball spacers. The PDLC samples were irradiated by a black-light lamp (ENF-240, Spectronics) at a light intensity of $1.6 \mathrm{~mW} /$ $\mathrm{cm}^{2}$ at $365 \mathrm{~nm}$ for $3 \mathrm{~min}$.

\subsection{Measurements}

Photo differential scanning calorimetry (photo-DSC) was used to examine the photopolymerization behavior. Photo-DSC thermograms were obtained using a photocalorimeter (TA5000, Thermal Analysis) under a nitrogen atmosphere. Photopolymerization was initiated using a black light lamp (B-14N, Spectronics) with a light intensity of $1.6 \mathrm{~mW} / \mathrm{cm}^{2}$ at $365 \mathrm{~nm}$. Samples weighing $1.0 \pm$ 0.1 (mean $\pm \mathrm{SD}$ ) $\mathrm{mg}$ were placed in uncovered aluminum pans, and a reference aluminum pan was left empty. All measurements were performed at room temperature. The area of the exothermic peak is proportional to the heat released as the thiol monomer is added across the double bonds to form single bonds during photopolymerization.

The thermal behaviors of the samples cured in photoDSC measurements were examined using DSC thermograms obtained with a DSC device (N-650, Scinco).

Scanning electron microscopy (SEM) was used to examine the PDLC morphology. The PDLC samples were freeze-fractured, soaked in hexane for $24 \mathrm{~h}$ to remove the LCs from the exposed surface, and then placed in a vacuum chamber for $24 \mathrm{~h}$ to remove the solvent.

Normal-normal spectral transmittance of PDLC cells was measured using an experimental setup comprising a halogen lamp (AvaLight-HAL, Avantes) and a CCD detector (AvaSpec-2048, Avantes). The transmittance spectra were obtained at a collection angle of $\pm 3^{\circ}$. The transmittance value at $650 \mathrm{~nm}$ was recorded from the transmittance-voltage curve.

\section{Results and Discussion}

The prepolymer formulations based on thiol-allyl ether chemistry were prepared to examine the effects of stoichiometric ratios of thiol to allyl ether monomer on the polymerization-induced phase separation, morphology, and electro-optical properties of PDLC films. In the polymerization mechanism of the thiol-ene system, the polymerization reaction is terminated by the stoichiometric imbalance, and there is no more thiol available to react with the ene or vice versa $[7,10]$. This approach was used 
to prepare prepolymer formulations with different stoichiometric ratios as listed in Table 1. The prepolymer formulations of the samples are expected to result in different degrees of monomer conversion. The numbers suffixed to "TE (the abbreviation of thiol-ene)" denote the molar percentage of the thiol group in the prepolymer formulation.

The photopolymerization behaviors of the prepolymers were investigated using photo-DSC. Photo-DSC experiments can provide kinetics data in which the measured heat flow can be converted directly to the ultimate percentage conversion and polymerization rate for a given formulation, with the data obtained reflecting the overall curing reaction of the sample [28-30]. Figure 2(a) shows the photo-DSC thermograms of the prepolymer samples, and Table 2 collects the parameters obtained from Figure 2(a), in which the $\Delta H_{\text {cure }}$ is the amount of heat released (the exotherm related to the conversion) and the $t_{\max }$ is the peak maximum (the time to attain the exotherm peak maximum related to the cure rate). As expected, the results reveal that as the molar ratio of thiol-ene mono-

Table 1. Prepolymer formulations with various molar ratios of thiol to allyl ether ([SH]:[AE]) monomers.

\begin{tabular}{cccc}
\hline Sample & 3-SH $(w t \%)$ & 4-AE $(w t \%)$ & {$[\mathrm{SH}]:[\mathrm{AE}]$} \\
\hline TE-35 & 30 & 70 & $35: 65$ \\
TE-40 & 35 & 65 & $40: 60$ \\
TE-45 & 40 & 60 & $45: 55$ \\
TE-50 & 45 & 55 & $50: 50$ \\
TE-60 & 55 & 45 & $60: 40$ \\
\hline
\end{tabular}

Table 2. Parameters obtained from the photo-DSC thermograms and thermal analysis of the prepolymer and PDLC samples.

\begin{tabular}{cccc}
\hline Sample & $\Delta H_{\text {cure }}{ }^{\mathrm{a}}(\mathrm{J} / \mathrm{g})$ & $t_{\max }{ }^{\mathrm{b}}(\mathrm{s})$ & $T_{\mathrm{g}}{ }^{\mathrm{c}}\left({ }^{\circ} \mathrm{C}\right)$ \\
\hline TE-35 & 158 & 5.0 & 1.6 \\
TE-40 & 190 & 4.4 & 4.5 \\
TE-45 & 207 & 4.2 & 6.9 \\
TE-50 & 237 & 4.0 & 7.4 \\
TE-60 & 203 & 4.2 & -8.4 \\
TE-35 + E7 & 179 & 9.6 & -24.8 \\
TE-40 + E7 & 205 & 8.8 & -22.9 \\
TE-45 + E7 & 215 & 8.2 & -13.4 \\
TE-50 + E7 & 239 & 7.6 & -12.3 \\
TE-60 + E7 & 214 & 8.6 & -26.5 \\
\hline
\end{tabular}

a. $\Delta H_{\text {cure }}$ : the amount of heat released. b. $t_{\max }$ : the time to attain the exotherm peak maximum. c. $T_{\mathrm{g}}$ : glass transition temperature of the polymer matrix.

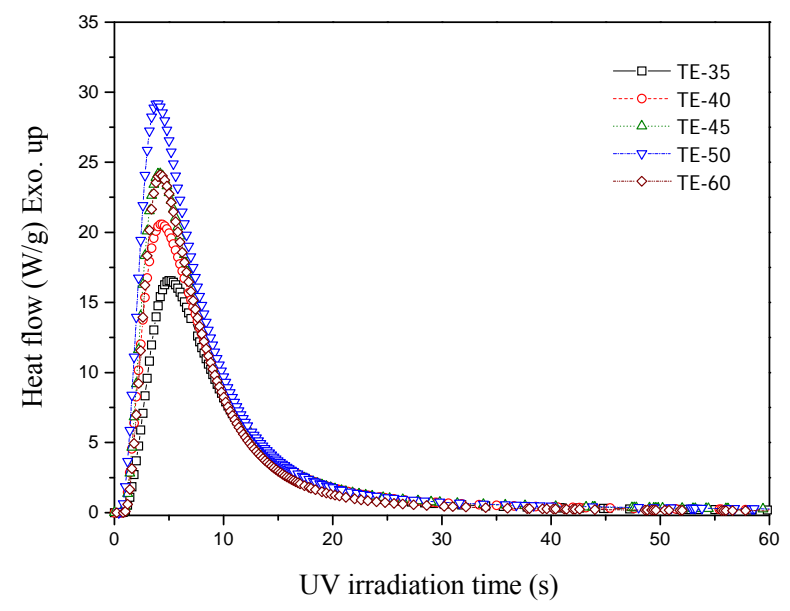

(a)

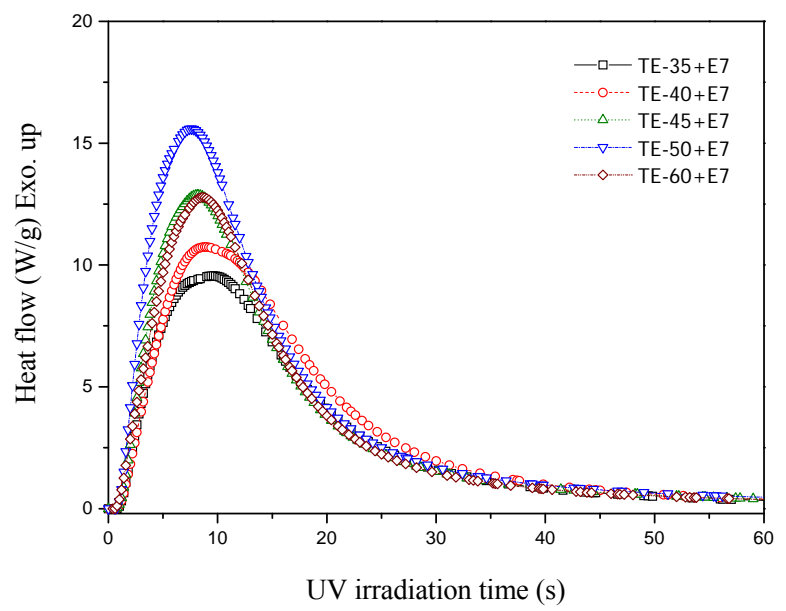

(b)

Figure 2. Photo-DSC thermograms of (a) the prepolymer samples and (b) the PDLC samples with various [SH]/[AE] values.

mers approaches the stoichiometric balance of $[\mathrm{SH}]:[\mathrm{AE}]$ $=50: 50$, both the exotherm and the photopolymerization rate increased to a maximum, and then decreased at the $60: 40$ molar ratio of $[\mathrm{SH}]:[\mathrm{AE}]$ due to the stoichiometric imbalance.

The photo-DSC thermograms of the PDLC samples are shown in Figure 2(b), and the $\Delta H_{\text {cure }}$ and the $t_{\max }$ are also listed in Table 2. To compare the photopolymerization behaviors of the PDLC samples with those of the prepolymer samples, the photo-DSC thermograms of the PDLC samples were normalized by the weights of the prepolymers. As shown in Table 2, the changes of the $\Delta H_{\text {cure }}$ and the $t_{\max }$ for the PDLC samples exhibited similar trend with those of the prepolymer samples. However, the PDLC samples gave higher exotherm than did the prepolymer samples, which is due to the effect of plasticization by the LC molecules. For the photopolymerization rate, the PDLC samples exhibited slower cure rates than those for the prepolymer samples probably due to 
the photopolymerization reaction being restricted by the LC molecules (a dilution effect) [1].

DSC experiments were carried out to investigate the thermal properties of the polymer matrix in PDLC network. First, DSC thermograms of the cured prepolymer samples are shown in Figure 3(a) and the obtained glass transition temperatures $\left(T_{\mathrm{g}}\right)$ of the polymer matrix are listed in Table 2. As the molar ratio of thiol-ene monomers approaches the stoichiometric balance of [SH]:[AE] $=50: 50$, the $T_{\mathrm{g}}$ values of the cured prepolymers increased to a maximum and then sharply decreased at the 60:40 molar ratio of $[\mathrm{SH}]:[\mathrm{AE}]$. Interestingly, the TE-60 sample showed relatively lower $T_{\mathrm{g}}$ than those for the other samples, which may be attributed to the chemical structure of 4-AE monomer with urethane groups. Hydrogen bonds could be formed by urethane groups and act as physical cross-links within the polymer matrix, probably contributing to increase the $T_{\mathrm{g}}$ value of the matrix. Therefore, it seems that the TE-60 sample containing less content of 4-AE showed the relatively low $T_{\mathrm{g}}$. And the

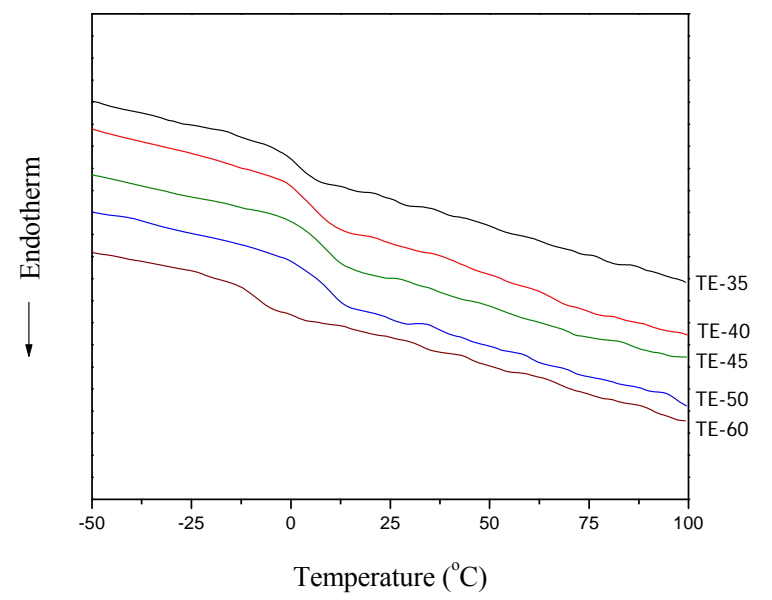

(a)

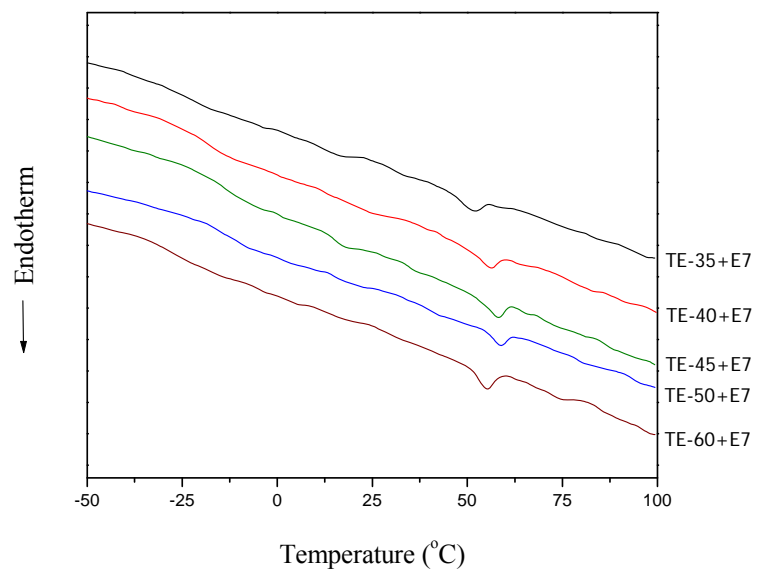

(b)

Figure 3. DSC thermograms of (a) the cured prepolymer samples and (b) the cured PDLC samples with various [SH]/ [AE] values. reason that the maximum $T_{\mathrm{g}}$ was obtained for TE-50 sample could be the matched stoichiometry by which the highest exotherm and degree of monomer conversion were achieved.

For the cured PDLC samples, as shown in Figure 3(b) and Table 2, the $T_{\mathrm{g}}$ values revealed similar behaviors with those of the cured prepolymer samples. However, the cured PDLC samples gave much lower $T_{\mathrm{g}}$ than did the cured prepolymer samples, which is probably due to the plasticizing effect by the LC molecules dissolved within the polymer matrix. Here, the endotherm peaks shown within the range $50^{\circ} \mathrm{C}-60^{\circ} \mathrm{C}$ in Figure 3(b) are the nematic-isotropic enthalpy of the LC droplets. Eventually, the $T_{\mathrm{g}}$ of thiol-ene-based PDLC systems was affected by the chemical structure of 4-AE monomer, stoichiometry, and the plasticizing effect by the LC molecules.

The relationship between the photopolymerization behaviors and morphology of the thiol-ene-based PDLC systems was examined using SEM. Figure 4 exhibits SEM photographs of the surface of the polymer matrix of the PDLC samples with different ratios of [SH]:[AE]. The figure clearly shows that the largest LC droplet size was obtained for TE-35 + E7 and the smallest for TE-50 $+\mathrm{E} 7$, which is probably related to the photopolymerization rate. The slow photocure rate of TE-35 + E7 would prolong the phase separation between the LC molecules and the prepolymer, leading to the largest LC droplet size. Presumably, the opposite phenomenon occurred in TE-50 + E7.

To investigate the relationship between the morphological and electro-optical properties of thiol-ene-based PDLC samples, the voltage dependencies of the transmittance of PDLC samples are shown in Figure 5. When the applied voltage increases, the transmittance of the PDLC samples reaches the saturation level $T_{\mathrm{s}}$. Figure 6 plots the threshold voltage $\left(V_{\mathrm{th}}\right)$ and the driving voltage $\left(V_{\mathrm{dr}}\right)$ as functions of the thiol concentration. Here, $V_{\text {th }}$ and $V_{\mathrm{dr}}$ are defined as the electric fields required for the transmittance to reach 10 and $90 \%$ of $T_{\mathrm{s}}$, respectively. As the thiol content increased, both $V_{\text {th }}$ and $V_{\mathrm{dr}}$ decreased obviously up to the stoichiometric balance of [SH]:[AE] $=50: 50$, and then increased at the 60:40 molar ratio of $[\mathrm{SH}]:[\mathrm{AE}]$. These behaviors are strongly influenced by the morphological properties with different LC droplet size $[1,2]$. The smaller the LC droplet size, the higher the $V_{\text {th }}$ and $V_{\mathrm{dr}}$ and the lower the $T_{\mathrm{s}}$. As a result, TE-50 + E7 sample with the smallest LC droplet size gave the highest threshold, driving voltages and lowest saturation transmittance because the orientation of LC molecules gets difficulty in small droplets.

\section{Conclusion}

Tetrafunctional allyl ether monomer (4-AE) was synthe- 


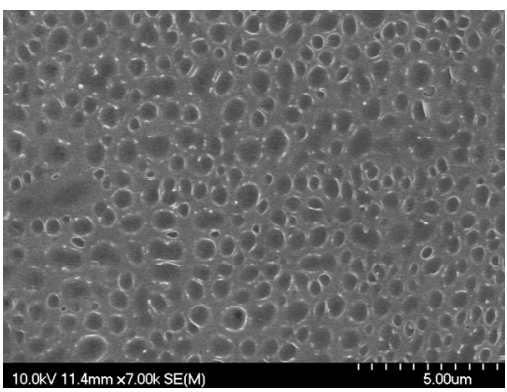

(a)

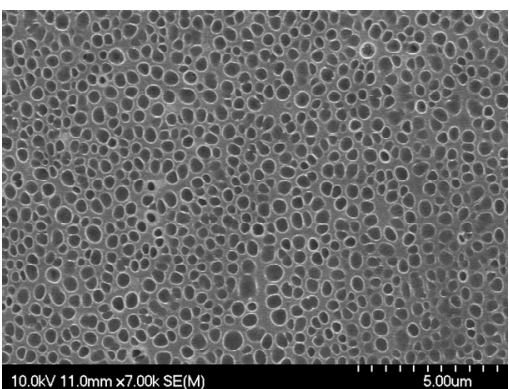

(b)

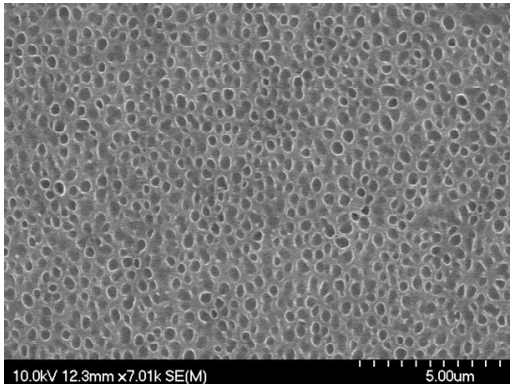

(c)

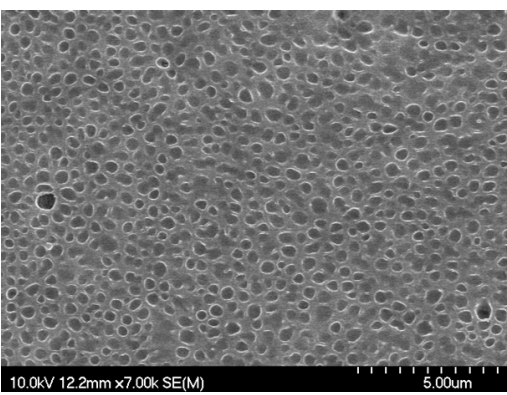

(d)

Figure 4. SEM micrographs of PDLC samples with various [SH]/[AE] values: (a) TE-35+E7, (b) TE-40+E7, (c) TE-50+ E7, and (d) TE-60+E7.

sized, and the effects of the different stoichiometric ratios of trimethylolpropane tris-(3-mercaptopropionate) (3-SH) to 4-AE on the photopolymerization behavior, morphology, and electro-optical properties of thiol-ene-based PDLC systems have been elucidated using photo-DSC, DSC, SEM, and electro-optical measurements. PhotoDSC and DSC analyses revealed that the PDLC sample containing $45 \mathrm{wt} \% 3-\mathrm{SH}$ and $45 \mathrm{wt} \%$ 4-AE gave the

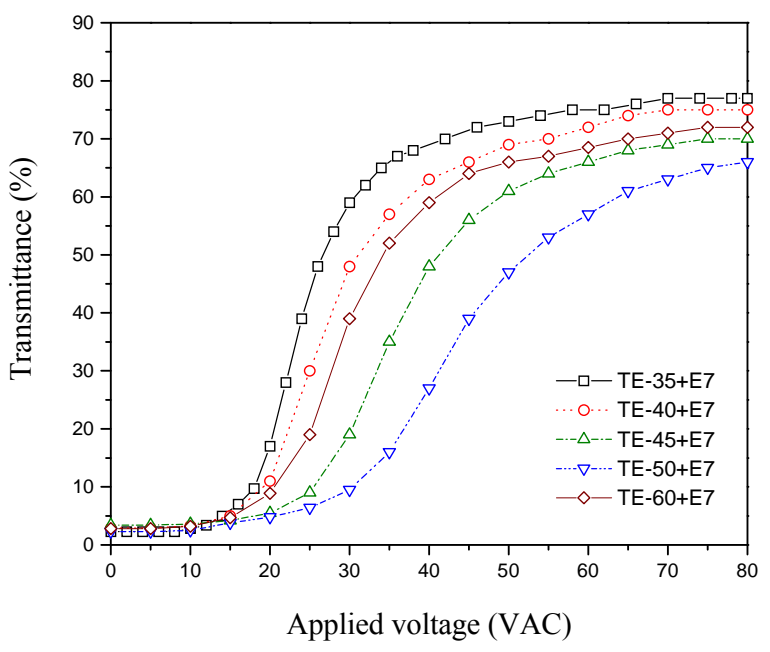

Figure 5. Transmittance of PDLC samples as functions of applied voltage.

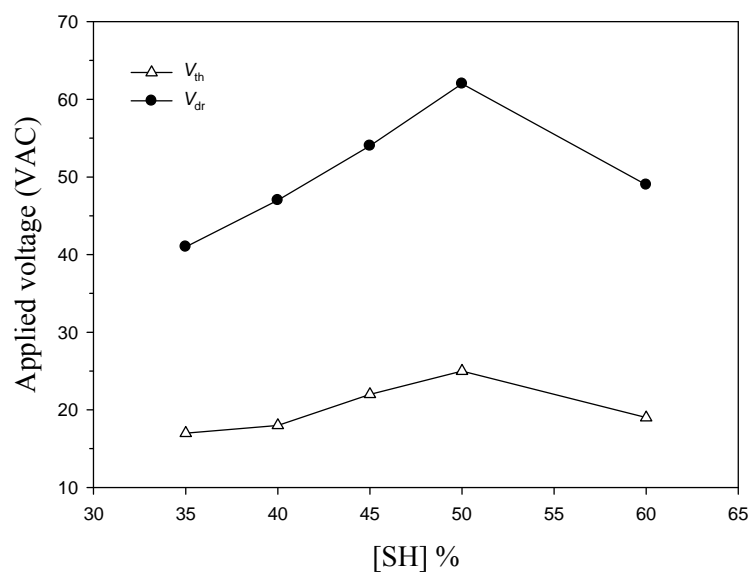

Figure 6. The thiol concentration dependence of $V_{\text {th }}$ and $V_{\mathrm{dr}}$.

highest exotherm, the fastest photocure rate, and the highest $T_{\mathrm{g}}$ due to the matched stoichiometry. The morphological observations showed that the maximum LC droplet size was obtained for the PDLC sample with 30 $\mathrm{wt} \% 3-\mathrm{SH}$ and the minimum for the sample with $45 \mathrm{wt} \%$ 3 -SH due to the different curing rate and phase separation behavior. The electro-optical measurements revealed that the PDLC sample with the matched molar ratio and smallest LC droplet size gave the highest threshold, driving voltages and lowest saturation transmittance because the orientation of LC molecules got difficulty in small droplets. Lastly, it was found that the stoichiometric ratios of 3-SH to 4-AE played a key role in controlling the photocure rate, phase separation rate, microstructures of LC droplets, and electro-optical properties of thiol-ene-based PDLC systems.

\section{REFERENCES}

[1] P. S. Drzaic, "Liquid Crystal Dispersions," World Scien- 
tific, Singapore, 1995.

http://dx.doi.org/10.1142/2337

[2] J. W. Doane, "Polymer Dispersed Liquid Crystal Displays," In: B. Bahadur, Ed., Liquid Crystals: Applications and Uses, World Scientific, Singapore, 1990, pp. 361-394. http://dx.doi.org/10.1142/9789814368278_0010

[3] N. Vaz, G. W. Smith and G. P. Montgomery, "A Light Control Film Composed of Liquid Crystal Droplets Dispersed in a UV-Curable Polymer," Molecular Crystals and Liquid Crystals, Vol. 146, No. 1, 1987, pp. 1-15. http://dx.doi.org/10.1080/00268948708071798

[4] B. K. Kim, Y. H. Cho and J. S. Lee, "Effect of Polymer Structure on the Morphology and Electro-Optic Properties of UV Curable PNLCs," Polymer, Vol. 41, No. 4, 2000, pp. 1325-1335.

http://dx.doi.org/10.1016/S0032-3861(99)00282-7

[5] D. Nwabunma and T. Kyu, "Phase Behavior, Photopolymerization, and Morphology Development in Mixtures of Eutectic Nematic Liquid Crystal and Photocurable Monomer," Polymer, Vol. 42, No. 2, 2001, pp. 801-806. http://dx.doi.org/10.1016/S0032-3861(00)00368-2

[6] T. Fujisawa, M. Hayasi, H. Nakada, Y. Tani and M. Aizawa, "An Analysis of Photo-Polymerization Induced Phase Separation Process in Liquid Crystal/Polymer Composite Films," Molecular Crystals and Liquid Crystals, Vol. 366, No. 1, 2001, pp. 107-116. http://dx.doi.org/10.1080/10587250108023953

[7] A. F. Jacobine, “Thiol-Ene Polymerization," In: J. P. Fouassier and J. F. Rabek, Eds., Radiation Curing in Polymer Science and Technology, Vol. III, Elsevier Applied Science, New York, 1993, pp. 219-268.

[8] B. K. Kim, S. H. Kim and J. C. Song, "Polymer Network Liquid Crystals from UV Curable Polyurethane Acrylate," Polymer, Vol. 39, No. 24, 1998, pp. 5949-5959. http://dx.doi.org/10.1016/S0032-3861(98)00043-3

[9] W. Zhang, J. Lin, T. Yu, S. Lin and D. Yang, "Effect of Electric Field on Phase Separation of Polymer Dispersed Liquid Crystal," European Polymer Journal, Vol. 39, No. 8, 2003, pp. 1635-1640. http://dx.doi.org/10.1016/S0014-3057(03)00074-0

[10] C. E. Hoyle, T. Y. Lee and T. Roper, "Thiol-Enes: Chemistry of the Past with Promise for the Future," Journal of Polymer Science Part A: Polymer Chemistry, Vol. 42, No. 21, 2004, pp. 5301-5338. http://dx.doi.org/10.1002/pola.20366

[11] B. G. Wu, J. H. Erdmann and J. W. Doane, "PDLC Shutters: Where Has This Technology Gone?" Liquid Crystals, Vol. 33, No. 11, 2006, pp. 1315-1322.

[12] J. A. Jung, B. K. Kim and J. C. Kim, "Effect of Oligomeric Surface Modifying Agent on Electro-Optical Properties of Polymer Dispersed Liquid Crystal," European Polymer Journal, Vol. 42, No. 10, 2006, pp. 2667-2671. http://dx.doi.org/10.1016/j.eurpolymj.2006.05.004

[13] S. H. Hwang, K. J. Yang, S. H. Woo, B. D. Choi, E. H. Kim and B. K. Kim, "Preparation of Newly Designed Reverse Mode Polymer Dispersed Liquid Crystals and its Electro-Optic Characteristics," Molecular Crystals and Liquid Crystals, Vol. 470, No. 1, 2007, pp. 163-171. http://dx.doi.org/10.1080/15421400701493653
[14] V. Rachet, P. Feneyrou, B. Loiseaux, R. L. Barny, J. P. Huignard and U. Maschke, "Electro-Optical Response of Thiol-Ene Based PDLC," Molecular Crystals and Liquid Crystals, Vol. 421, No. 1, 2004, pp. 165-174. http://dx.doi.org/10.1080/15421400490501761

[15] A. F. Senyurt, G. Warren, J. B. Whitehead Jr. and C. E. Hoyle, "Matrix Physical Structure Effect on the ElectroOptic Characteristics of Thiol-Ene Based H-PDLC Films," Polymer, Vol. 47, No. 8, 2006, pp. 2741-2749. http://dx.doi.org/10.1016/j.polymer.2006.02.049

[16] O. Yaroshchuk, F. Elouali and U. Maschke, "Control of Phase Separation and Morphology of Thiol-Ene Based PDLCs by Curing Light Intensity," Optical Materials, Vol. 32, No. 9, 2010, pp. 982-989. http://dx.doi.org/10.1016/j.optmat.2010.02.017

[17] J. W. Seok, H. S. Ryu, H. J. Seo, W. S. Kim, D. H. Lee, K. E. Min, K. H. Seo, I. K. Kang and L. S. Park, "Effect of Oligomeric Photoinitiator on the Preparation and Electro-Optical Property of Polymer-Dispersed Liquid Crystal," Optical Materials, Vol. 21, No. 1-3, 2003, pp. 633636. http://dx.doi.org/10.1016/S0925-3467(02)00213-6

[18] J. J. Koo, Y. S. No, C. W. Jeon and J. H. Kim, "Improvement of Electro-Optic Properties in PDLC Device by Using New Cross-Linker for the Control of the Contrast Ratio, Response Time and Driving Voltage," Molecular Crystals and Liquid Crystals, Vol. 491, No. 1, 2008, pp. 58-66.

http://dx.doi.org/10.1080/15421400802328899

[19] Y. S. No, J. H. Kim, C. W. Jeon and S. H. Park, "Temperature Stability of Electro-Optic Properties of Polymer Dispersed Liquid Crystal with Different Crosslinking Monomer in PN393 Base Pre-Polymer," The Korean Journal of Chemical Engineering, Vol. 25, No. 1, 2008, pp. 181-184. http://dx.doi.org/10.1007/s11814-008-0033-2

[20] L. Wenbo, J. Mengjun, D. Xiaokang, L. Bofu, H. Wei, C. Hui, Y. Zhou and Y. Huai, "Studies on Electro-Optical Properties of Polymer Matrix/LC/SiO 2 Nanoparticles Composites," Journal of Applied Polymer Science, Vol. 111, No. 3, 2009, pp. 1449-1453.

http://dx.doi.org/10.1002/app.28874

[21] Y. S. No and C. W. Jeon, "Effect of Alignment Layer on Electro-Optic Properties of Polymer-Dispersed Liquid Crystal Displays," Molecular Crystals and Liquid Crystals, Vol. 513, No. 1, 2009, pp. 98-105. http://dx.doi.org/10.1080/15421400903195759

[22] S. Park and J. W. Hong, "Polymer Dispersed Liquid Crystal Film for Variable-Transparency Glazing," Thin Solid Films, Vol. 517, No. 10, 2009, pp. 3183-3186. http://dx.doi.org/10.1016/j.tsf.2008.11.115

[23] K. Miki, C. Hui, M. Qingyong, L. Huijing, W. Ding, L. Fasheng and Y. Huai, "The Influence of Crosslinking Agents on the Morphology and Electro-Optical Performances of PDLC Films," Journal of Applied Polymer Science, Vol. 117, No. 6, 2010, pp. 3434-3440.

[24] G. P. Montgomery and N. A. Vaz, "The Relationship between Formation Kinetics and Microdroplet Size of Epoxy-Based Polymer-Dispersed Liquid Crystals," Liquid Crystals, Vol. 3, No. 5, 1988, pp. 543-571. http://dx.doi.org/10.1080/02678298808086401 
[25] T. J. White, L. V. Natarajan, T. J. Bunning and C. A. Guymon, "Contribution of Monomer Functionality and Additives to Polymerization Kinetics and Liquid Crystal Phase Separation in Acrylate-Based Polymer-Dispersed Liquid Crystals (PDLCs)," Liquid Crystals, Vol. 34, No. 12, 2007, pp. 1377-1385. http://dx.doi.org/10.1080/02678290701663936

[26] D. Hatice, M. Scott, K. Namil, H. Jun, K. Thein, V. N. Lalgudi, P. T. Vincent and J. B. Timothy, "Kinetics of Photopolymerization-Induced Phase Separation and Morphology Development in Mixtures of a Nematic Liquid Crystal and Multifunctional Acrylate," Polymer, Vol. 49, No. 2, 2008, pp. 534-545. http://dx.doi.org/10.1016/j.polymer.2007.11.039

[27] J. Zou and J. Fang, "Adhesive Polymer-Dispersed Liquid Crystal Films," Journal of Materials Chemistry, Vol. 21, No. 25, 2011, pp. 9149-9153. http://dx.doi.org/10.1039/c1jm10628g

[28] S. C. Clark, C. E. Hoyle, S. Jönsson, F. Morel and C.
Decker, "Photopolymerization of Acrylates Using NAliphaticmaleimides as Photoinitiators," Polymer, Vol. 40, No. 18, 1999, pp. 5063-5072. http://dx.doi.org/10.1016/S0032-3861(98)00734-4

[29] J. D. Cho, H. T. Ju and J. W. Hong, "Photocuring Kinetics of UV-Initiated Free-Radical Photopolymerizations With and Without Silica Nanoparticles," Journal of Polymer Science Part A: Polymer Chemistry, Vol. 43, No. 3, 2005, pp. 658-670.

http://dx.doi.org/10.1002/pola.20529

[30] J. D. Cho, H. T. Ju, Y. S. Park and J. W. Hong, "Kinetics of Cationic Photopolymerizations of UV-Curable EpoxyBased SU8-Negative Photoresists with and without Silica Nanoparticles," Macromolecular Materials and Engineering, Vol. 291, No. 9, 2006, pp. 1155-1163. http://dx.doi.org/10.1002/mame.200600124 